\title{
Mechanical properties of hydrogenated bilayer graphene
}

\author{
R. C. Andrew, ${ }^{1, *}$ R. E. Mapasha, ${ }^{1, \dagger}$ and N. Chetty ${ }^{1,2, *}$ \\ ${ }^{1}$ Physics Department, University of Pretoria, Pretoria 0002, South Africa \\ ${ }^{2}$ National Institute for Theoretical Physics, Johannesburg, 2000, South Africa
}

(Dated: August 15, 2013)

\begin{abstract}
Using first principles methods, we study the mechanical properties of monolayer and bilayer graphene with 50\% and $100 \%$ coverage of hydrogen. We employ the vdW-DF, vdW-DF-C09 $x$ and vdW-DF2-C09 van der Waals functionals for the exchange correlation interactions that give significantly improved interlayer spacings and energies. We also use the PBE form for the generalized gradient corrected exchange correlation functional for comparison. We present a consistent theoretical framework for the in-plane layer modulus and the out-of-plane interlayer modulus and we calculate, for the first time, these properties for these systems. This gives a measure of the change of the strength properties when monolayer and bilayer graphene are hydrogenated. As well as comparing the relative performance of these functionals in describing hydrogenated bilayered graphenes, we also benchmark these functionals in how they calculate the properties of graphite.
\end{abstract}

PACS numbers: 61.48.Gh,62.20.de,64.10.+h

\section{INTRODUCTION}

Monolayer and bilayer graphene continue to be extensively studied because of their potential applications in nanoscale devices. ${ }^{1-8}$ It is well-known that these materials lack an intrinsic electronic band gap ${ }^{3}$ which hinders their application for nanoelectronics. As a result, substantial efforts have been made towards opening and controlling the band gap, and several methods have been developed. Applying an electric field between the layers in bilayer graphene is one possible option. ${ }^{9}$ Another promising technique is the adsorption of foreign atoms. ${ }^{10-25}$ It must be noted that not all foreign atoms open up a bandgap, e.g, the adsorption of lithium atoms makes these structures metallic. ${ }^{26-31}$ Recently, the use of hydrogen adatoms has been studied and theoretical investigations show that these hydrogenated materials are wide bandgap systems, ${ }^{13,24,32-34}$ a result supported by experiment. ${ }^{25}$

Although the structural and electronic properties of hydrogenated monolayer and bilayer graphene have been extensively studied, the mechanical response properties of these materials require further investigation. When hydrogen bonds with carbon in a graphene sheet, the translation symmetry of the $\mathrm{sp}^{2} \mathrm{C}-\mathrm{C}$ bonds breaks, leading to the carbon atom having $\mathrm{sp}^{3}$ hybridization due to wavefunction overlap. In previous studies, ${ }^{32 ?, 33}$ it was found that hydrogen marginally increases or decreases the interlayer spacing of bilayer graphene depending on its position on the layers. It is expected that the adsorption of hydrogen will affect the mechanical properties such as the material's resilience to in-plane stretching and outof-plane compression.

In order to study the in-plane mechanical properties of 2D materials, Andrew et al. ${ }^{35}$ introduced a 2D equation of state (EOS) that equated the isotropic in-plane 2D pressure (the force per unit length $\mathcal{F}$ ) to the surface area. This also gave the material's resilience to in-plane stretching (the layer modulus $\gamma$ ) as one of its fit parameters. Using this equation, bilayer graphene was studied for its strength properties as well as the in-plane 2D pressure response of its layer modulus.

In this work, we present the in-plane mechanical properties of hydrogenated monolayer and bilayer graphene using this 2D EOS. We also test and compare a set of recently developed non-local corrected exchange correlation functionals since they are known to better describe the interlayer interaction. The resistance to out-of-plane compression is also studied through a new property, namely the interlayer modulus $\beta$. This modulus can be obtained by fitting out-of-plane surface pressure data over a set of interlayer distances to an out-ofplane EOS.

This paper is organized as follows: In Section II we present the theoretical framework for the non-local corrected functionals, the in-plane and out-of-plane moduli with their respective equation of states, and a brief summary of the computational methodology. In Section III, we apply our methods to single and bilayer hydrogenated graphene, and we draw our concluding remarks in Section IV.

\section{THEORETICAL FRAMEWORK}

\section{A. Non-local corrected functionals}

The interaction energy curve as a function of separation distance for a system that includes non-local van der Waals forces is characterized by an exponentially rising repulsive wall on the compressive side of the equilibrium separation with a slow decaying attractive van der Waals tail on the expansion side. Many methods have been implemented in order describe this interaction which is found in molecular, sparse and layered systems.

The semi-empirical force-field correction of Grimme ${ }^{36}$ includes damped atom-pairwise dispersion corrections of the form $C_{6} R^{-6}$. Other "universal" functionals have also been developed which, qualitatively, have a sum of contributions of the same form in the asymptotic region of the interaction energy. Two such universal functionals are vdW-DF developed by Dion et al. ${ }^{37}$ and vdW-DF2 developed by Lee et al. ${ }^{38}$ The functional of Lee et al. was presented as an improvement over vdW-DF and has weaker attraction in the asymptotic region with smaller calculated $C_{6}$ values. It generally gives better equilibrium spacings and binding energies. 
Both functionals are constructed in the same manner. The correlation energy part of these functionals is divided into two parts viz., local and non-local $(n l)$ correlations. The local energy correlation employs LDA to account for short-range interactions, whereas the non-local energy correlation is formulated as

$$
E_{c}^{n l}=\frac{1}{2} \iint n(\mathbf{r}) n\left(\mathbf{r}^{\prime}\right) \phi\left(q_{1}, q_{2}, r_{12}\right) d^{3} \mathbf{r} d^{3} \mathbf{r}^{\prime}
$$

where the kernel $\phi$ is a function of $r_{12}=\left|\mathbf{r}-\mathbf{r}^{\prime}\right|$ and $q_{1}$ and $q_{2}$ are functions of a universal function $q_{0}[n(\mathbf{r}),|\nabla n(\mathbf{r})|]$ evaluated at the points $\mathbf{r}$ and $\mathbf{r}^{\prime}$. The full potential approximation (FPA) is then applied to $E_{c}^{n l}$ in order to avoid double counting for short-range interactions. The exchange energy is obtained from the revised Perdew Burke and Enzerhof ${ }^{39}$ exchange term $E_{x}^{r e v P B E}$ in the case of vdW-DF, and the Cooper ${ }^{40}$ exchange term $E_{x}^{C 09}$ in the cases of vdW-DF-C09 $x$ and vdW-DF2-C09. The reason for the use of the Cooper exchange is to reduce the short-range repulsion due to the revPBE functional which leads to underbinding with overestimated equilibrium separations.

In 2006, it was found by Dobson et al. ${ }^{41}$ that the sum of $C_{6} R^{-6}$ terms used in many van der Waals corrected methods incorrectly predicts the proper energy power laws for a variety of systems. Although it was felt that "universal" schemes would display valid asymptotic results for most systems, they would fail for anisotropic nanostructures such as the hydrogenated weakly bound layered systems studied here. For instance, they predicted that for bilayer graphene the asymptotic attractive energy is of the form $-C_{3} D^{-3}$ as opposed to the $D^{-4}$ behavior predicted by the sum of $C_{6} R^{-6}$ terms. Since the random phase approximation ${ }^{42}$ (RPA) does not rely on $R^{-6}$ contributions, it is commonly used as a benchmark in order to validate calculated results for van der Waals corrected functionals. ${ }^{41,43,44}$

For the reasons given above, we have chosen to investigate the vdW-DF, vdW-DF-C09 $x$ and vdW-DF2-C09 ${ }_{x}$ functionals ${ }^{37,40,45-47}$ in how they describe hydrogenated graphene systems as well as bulk graphite. We also use the PBE form for the generalized gradient corrected exchange correlation functional ${ }^{45}$ for comparison.

\section{B. In-plane 2D layer modulus}

Given the in-plane 2D pressure (force per unit length) is defined as

$$
\mathcal{F}=-\frac{\partial E}{\partial A}
$$

and the resistance to in-plane stretching (the layer modulus) is defined as

$$
\gamma=-A \frac{\partial \mathcal{F}}{\partial A}
$$

the $2 \mathrm{D} \mathrm{EOS}^{35}$ that relates the isotropic in-plane 2D pressure to the surface area is given by

$$
\mathcal{F}(A)=-2 \gamma_{0}\left[\epsilon+\left(1-\gamma_{0}^{\prime}\right) \epsilon^{2}+\frac{2}{3}\left(\left(1-\gamma_{0}^{\prime}\right)\left(2-\gamma_{0}^{\prime}\right)+\gamma_{0} \gamma_{0}^{\prime \prime}\right) \epsilon^{3}\right]
$$

where

$$
\epsilon=\frac{1}{2}\left(1-A_{0} / A\right)
$$

and $A_{0}, \gamma_{0}, \gamma_{0}^{\prime}$ and $\gamma_{0}^{\prime \prime}$ are the equilibrium values for the unit cell area, layer modulus, the force per unit length first derivative and second derivative of the layer modulus at $\mathcal{F}=0$ respectively. This EOS can be fitted to isotropic force per unit length values calculated over a range of area values. It has an advantage over the energy EOS used by Andrew et $\mathrm{al}^{35}$. Whereas there was a slight ambiguity in the range of areas that had to be used for that EOS, the range of areas to be used for the pressure EOS is fully defined by the full positive pressure range from the expected zero in-plane $2 \mathrm{D}$ pressure value to the expected maximum intrinsic breaking stress. Typically, the equilibrium in-plane distances increase by $20 \%$ over this range.

The $\gamma$-related fit parameters define the in-plane 2D pressure response of the layer modulus near the equilibrium point where

$$
\gamma=\gamma_{0}+\gamma_{0}^{\prime} \mathcal{F}+\frac{1}{2} \gamma_{0}^{\prime \prime} \mathcal{F}^{2}
$$

\section{Out-of-plane interlayer modulus}

In their study of graphite, Hanfland et al. ${ }^{48}$ defined the linear bulk modulus in the $c$ axis as

$$
\beta_{c}=-c \frac{\partial P}{\partial c}
$$

where $c$ is the lattice constant and $P$ the bulk pressure. In terms of the elastic constants, this modulus is

$$
\beta_{c}=C_{33}+C_{13}
$$

Similarly, we can define, for finite layered 2D materials, the interlayer modulus

$$
\beta \equiv \beta_{d}=-d \frac{\partial p}{\partial d}
$$

where $d$ is the interlayer distance (defined as the vertical distance between the lowest atom in an upper layer and the highest atom in the lowest layer) and $p$ is the pressure on the external surfaces of the two outermost layers. This modulus represents the material's resistance to out-of-plane compression.

The out-of plane EOS that relates the surface pressure to the interlayer distance is

$$
p(d)=-2 \beta_{0}\left[\epsilon+\left(1-\beta_{0}^{\prime}\right) \epsilon^{2}+\frac{2}{3}\left(\left(1-\beta_{0}^{\prime}\right)\left(2-\beta_{0}^{\prime}\right)+\beta_{0} \beta_{0}^{\prime \prime}\right) \epsilon^{3}\right]
$$


where

\section{RESULTS AND DISCUSSION}

$$
\epsilon=\frac{1}{2}\left(1-d_{0} / d\right)
$$

and $d_{0}, \beta_{0}, \beta_{0}^{\prime}$ and $\beta_{0}^{\prime \prime}$ are the equilibrium values for the interlayer distance, interlayer modulus, the surface pressure first derivative and second derivative of the interlayer modulus at $p=0$, respectively. This EOS can be fitted to surface pressure values calculated over a range of interlayer distances.

These fit parameters define the surface pressure response of the interlayer modulus near the equilibrium point where

$$
\beta=\beta_{0}+\beta_{0}^{\prime} p+\frac{1}{2} \beta_{0}^{\prime \prime} p^{2}
$$

\section{Computational details}

All calculations were done within the framework of density functional theory ${ }^{49}$ (DFT) using the projector augmentedwave $^{50}$ (PAW) formalism as implemented in the QuANTUM ESPRESSO package. ${ }^{51}$ We used the GGA-PBE ${ }^{45}$, vdW-DF, vdW-DF-C09 $x$ and vdW-DF2-C09 functionals. For the monolayer and bilayer structures, a Monkhorst-Pack ${ }^{52}$ grid of $10 \times 10 \times 1$ was chosen since testing showed it gave an error in the total energy less than $1 \mathrm{meV}$. A grid size of $9 \times 9 \times 5$ was chosen for graphite for the same reason.

The total energy calculations were converged to within $10^{-8}$ Ryd and a Methfessel-Paxton distribution function ${ }^{53}$ with a smearing parameter of $0.05 \mathrm{Ry}$ was used to integrate the bands at the Fermi level. In all cases, a kinetic energy cutoff of 40 Ryd was used. The vdW stress components were calculated using the method of Sabatini et al. ${ }^{54}$ as implemented in PWscf. All atomic forces during relaxation were converged to within 0.0001 a.u

For all structures, a $1 \times 1$ in-plane unit cell was used. The cell height was chosen to create an adequate vacuum layer to isolate the structure. For all monolayer structures and pristine bilayer graphene, the cell height was set to $c=15 \AA$ in order to prevent spurious interactions between cells repeating perpendicular to the layer plane. A larger height of $30 \AA$ was used for the hydrogenated bilayer materials. To determine the interlayer modulus for pristine bilayer graphene, the cell height was also set to $30 \AA$.

For the calculation of the in-plane properties, the atoms were fully relaxed at each chosen area value. For the calculation of the out-of-plane properties of the bilayer materials, the in-plane 2D cell was fully relaxed along with the atomic positions while keeping the vacuum height and the vertical positions of the atoms defining the chosen interlayer distance fixed. For the calculation of the out-of-plane properties of graphite, the in-plane lattice constant was relaxed at each fixed $c$ value.

\section{A. Structures}

We investigate the mechanical properties of hydrogenated monolayer and bilayer graphene with both $50 \%$ and $100 \%$ coverages. These structures are shown in Fig. 1.

The top-view of monolayer graphene with $50 \%$ coverage [see Fig. 1a] shows that the hydrogen adatoms are attached to every other carbon atom. The bottom of the monolayer sheet is the same as for graphene indicating that only one side of this structure is hydrogenated. This structure is referred to in the tables as graphene $+\mathrm{H}(50 \%)$. In contrast, monolayer graphene with $100 \%$ coverage (namely, graphane) has a top surface with hydrogenation on every other atom [see Fig. 1b] with a bottom surface the same as that shown in Fig. 1a. This indicates hydrogen adatoms are attached to both sides of the sheet in an alternating pattern.

The hydrogenated bilayer graphene has the Bernal $A B$ stacking (as shown in Fig. 1c and Fig. 1d) as this is more energetically stable than simple $A A$ stacking. ${ }^{34}$ In bilayer graphene with $50 \%$ coverage, each layer has only $50 \%$ coverage with no hydrogen atoms between the layers. This is referred to in the tables as bilayer graphene $+\mathrm{H}(50 \%)$. Bilayer graphene with $100 \%$ coverage (namely, bilayer graphane) consists of two graphene sheets with hydrogen adsorption on both sides of each layer.

Table. I shows the equilibrium structural parameters obtained from the in-plane EOS fits. It can be seen from the values for the lattice constant $a_{0}$ that all four functionals describe similar in-plane covalent bonding. The non-local functionals containing the $C 09_{x}$ exchange term give results for graphene and bilayer graphene that are very close to the experimental value of $2.463 \AA$ obtained by Bosak et al. ${ }^{55}$ for graphite. The other two functionals, with their greater repulsive exchange terms, give slightly higher values that are greater than the experimental value.

For the hydrogenated structures, all four functionals give similar buckling parameters where the buckling parameter is defined as the vertical distance between the highest and the lowest carbons in a given layer. The values for bilayer graphene with $50 \%$ coverage are slightly higher due to the tetrahedral nature of the diamond-like interlayer bonding as seen in Fig. 1c.

\section{B. In-plane mechanical properties}

For all structures, Eqn. 4 was used to fit calculated inplane $2 \mathrm{D}$ pressure values for a range of areas using the four exchange-correlation functionals to obtain the fit parameters given in Table. I (all fits give the same $a_{0}$ parameter as the values obtained from geometric relaxation).

To obtain these fits, the isotropic in-plane 2D pressure is calculated from the stresses $\sigma_{x x}, \sigma_{y y}$ and the cell height $c$ as

$$
\mathcal{F}=\frac{c}{2}\left(\sigma_{x x}+\sigma_{y y}\right)
$$


This converts the bulk stresses acting on the sides of the cell into an in-plane 2D pressure that is independent of the cell height (provided there is a sufficient amount of vacuum).

A plot of the EOS fits for pristine bilayer graphene and and bilayer graphane are given in Fig. 2a. This shows that isotropic in-plane $2 \mathrm{D}$ pressure grows more negative as the structures are stretched. At a certain point, the trend reverses indicating the expected intrinsic breaking point for the structure at the resulting minimum. The plot shows that pristine bilayer graphene has the higher breaking stress (where $\sigma_{\text {int }}=$ $-\mathcal{F}_{\text {int }}$ ) of $64.3 \mathrm{Nm}^{-1}$ (compared to $41.7 \mathrm{Nm}^{-1}$ for bilayer graphane) and that it yields at a smaller area of 27.5 bohr $^{2}$ (compared to $29.4 \mathrm{bohr}^{2}$ for bilayer graphane). The relative breaking area (defined as $A_{\text {int }} / A_{0}$ ) is basically the same for both structures having a value of $1.46 \sim 1.47$.

From these fits, various trends in the behavior of each functional can be seen. It is evident that for all four functionals, the bilayer structures have greater resilience to in-plane stretching than the monolayer structures as indicated by their relatively higher values for $\gamma_{0}$. The hydrogenated structures are also less resilient than their pristine counterparts. For each structure, the $\gamma_{0}$ and $\sigma_{i n t}$ values show slight variation with the choice of functional with an increasing trend going from $\mathrm{vdW}$ $\mathrm{DF} \rightarrow \mathrm{GGA}-\mathrm{PBE} \rightarrow \mathrm{vdW}-\mathrm{DF} 2-\mathrm{C} 09_{x} \rightarrow \mathrm{vdW}-\mathrm{DF}-\mathrm{C} 09_{x}$.

Looking at the results for the linear and parabolic force per unit length derivative terms $\gamma_{0}^{\prime}$ and $\gamma_{0}^{\prime \prime}$ it is clear that, in general, vdW-DF gives the highest absolute values when compared to the other three functionals (except in the case of monolayer graphene with 50\% coverage where PBE gives the greatest negative value for $\gamma_{0}^{\prime \prime}$ ). For the unhydrogenated systems, the two $\mathrm{C} 09_{x}$ functionals give almost the same values for $\gamma_{0}^{\prime}$ and $\gamma_{0}^{\prime \prime}$ as compared with the PBE functional. Monolayer graphene with $50 \%$ coverage has $\gamma_{0}^{\prime}<4.20$ for all four functionals whereas $\gamma_{0}^{\prime}>4.20$ for all the other systems. The values for the relative intrinsic breaking area $A_{\text {int }} / A_{0}$ show that for each structure, the functionals all give more or less the same value.

In Table. II, we compare the parameters for a given structure with those for a corresponding reference structure. The LDA results of Andrew et al. ${ }^{35}$ demonstrated that the effect of layering in the case of graphene was an increase in $\gamma_{0}$ and $\sigma_{\text {int }}$ with a doubling of these values for bilayer graphene. The inplane 2D pressure response of the layer modulus (see Eqn. 6) also became more linear with $\gamma_{0}^{\prime \prime}$ having half the value of pristine graphene. All other parameters remained the same. This can be seen in Table. II in the case of bilayer graphene compared with graphene. For all four functionals, the ratios for $a_{0}, \gamma_{0}^{\prime}$ and $A_{\text {int }}$ are basically unity whereas those for $\gamma_{0}$ and $\sigma_{\text {int }}$ are doubled while $\gamma_{0}^{\prime \prime}$ is halved. This is indicative of weak interlayer interaction, and in the case of PBE it is revealed that there is practically no interlayer interaction.

The results of the hydrogenated monolayer graphene (compared with graphene) show that the adsorption of hydrogen increases the lattice constant by a factor of about 1.02 1.03. The value for $\gamma_{0}$ decreases by $0.64 \sim 0.68$ with that for $\sigma_{\text {int }}$ generally following the same trend. The values for $\gamma_{0}^{\prime \prime}$ show that $\gamma(\mathcal{F})$ for both these structures is more parabolic compared to monolayer graphene. This feature of buckled monolayer ma- terials has been observed theoretically in honeycomb Ge and $\mathrm{Si}$ where $\mathrm{Ge}$ had the greatest non-linear response due to its larger buckling parameter. ${ }^{35}$ Monolayer graphene with $50 \%$ coverage shows the greatest deviation from linear response having the highest increase in $\gamma_{0}^{\prime \prime}$ and a relative decrease in the linear term $\gamma_{0}^{\prime}$. This result is interesting since graphane has the larger buckling parameter and it is expected that this structure should have the greatest non-linear response. It is thought that this discrepancy is due to the fact that whereas graphane has symmetrical puckering due to adatoms being on either side of the monolayer, this is not the case for graphene with $50 \%$ coverage where all the hydrogen atoms are on one side. The ratios for $A_{\text {int }}$ are slightly greater than unity.

The results for the hydrogenated bilayer graphenes (compared with their monolayer counterparts) show that the effect of layering is largely dependent on the amount of interlayer bonding. Weak bonding gives results similar to the trends seen for bilayer graphene, and this is indeed the case for bilayer graphane. The covalent bonding in bilayer graphene with $50 \%$ coverage yields results that deviate from this expected trend. For instance $\gamma_{0}$ is increased by $\sim 1.9$ for all four functionals and $\sigma_{\text {int }}$ by $\sim 1.8$. The linear term $\gamma_{0}^{\prime}$ is increased by $1.12 \sim 1.23$ instead of remaining the same and the parabolic term $\gamma_{0}^{\prime \prime}$ for the vdW functionals is decreased by $0.41 \sim 0.48$. The covalent interlayer bonding weakens the in-plane covalent bonding suppressing the doubling effect for $\gamma_{0}$. It also suppresses the increased linearity usually seen with increasing layers though it does increase the linear term which may compensate for this. As with the hydrogenated single layer graphene, the ratios for $A_{i n t}$ are slightly greater than unity.

\section{Out-of-plane mechanical properties}

\section{Graphite}

Since graphite has been extensively studied both experimentally ${ }^{55,56}$ and theoretically, ${ }^{43,44}$ we have included calculations of its out-of-plane properties using the EOS of Hanfland et al. ${ }^{48}$ The binding energy was also calculated by taking the difference between the asymptotic interaction energy at large separation and that at the equilibrium distance. The results are shown in Table. III.

The results show that of the four functionals considered, vdW-DF2-C09 $x$ gives a value for the interlayer distance that is closer to the experimental value of $3.356 \AA .{ }^{55}$ The functional vdW-DF- $C 09_{x}$ gives a very similar value which is due to the fact that is has the same exchange term which is known to correct for over-estimation. ${ }^{40} \mathrm{PBE}$ is well above this value due to its greater repulsive nature. This is also true for vdW-DF which contains the revPBE exchange term. As a benchmark, vdW-DF-C $09_{x}$ and vdW-DF2-C09 $x$ compare very well with the RPA value calculated by Lebègue et al. ${ }^{43}$

The calculated binding energies per atom show that vdWDF and vdW-DF2-C09x, with basically the same value of $55 \mathrm{meV} / \mathrm{atom}$, are closer to the experimental value of $52 \mathrm{meV} /$ atom measured by Zacharia et al. ${ }^{56}$ and the benchmark RPA value of $48 \mathrm{meV} /$ atom calculated by Lebègue et 
al. $^{43}$. The functional vdW-DF-C $09_{x}$ overestimates the binding energy which is interesting since it only differs from vdW-DF in that it has a different exchange term. This shows that the exchange term which sets the position of the repulsive wall in the interaction energy curve, also affects the position of the energy minimum relative to the van der Waals tail.

The linear bulk modulus was also calculated for each functional over a range of pressures from approximately 0 to $9 \mathrm{GPa}$ in the $c$ direction. The relationship between this modulus and the elastic constants for a material are given by Eqn. 8 . For graphite, this value is $C_{33}$ since $C_{13}$ is quite close to zero. ${ }^{55}$ The values show an increasing trend from $\mathrm{PBE} \rightarrow \mathrm{vdW}$ $\mathrm{DF} \rightarrow \mathrm{vdW}-\mathrm{DF} 2-C 09_{x} \rightarrow \mathrm{vdW}-\mathrm{DF}-C 09_{x}$. The experimental measurements of Bosak et al. give a value of $38.7 \mathrm{GPa}$ which is close to the value of $38.3 \mathrm{GPa}$ given by vdW-DF2-C09. Lebègue et al. calculated a RPA value of $36 \mathrm{GPa}$ and although the in-plane lattice constant was not optimized at each $c$ value as was done in this study, we believe that it is still a good benchmark value.

These results show that for layered graphite, vdW-DF2$C 09_{x}$ give the most accurate values of the three van der Waals corrected functionals considered.

\section{Bilayered structures}

In order to use Eqn. 10 for the bilayered structures, the surface pressure $p$ acting on the outermost layers has to be defined in terms of the calculated out-of-plane stress $\sigma_{z z}$ and the periodic cell dimensions. One such definition is

$$
p=c\left(\frac{u+v}{A}\right) \sigma_{z z}
$$

where $c$ is the cell height, $u$ and $v$ are the lengths of the inplane spanning vectors and $A$ is the in-plane cell area. We have checked that this definition gives essentially constant $p$ values for a given layered structure independent of the cell height (provided there is a sufficient amount of vacuum).

Using this definition, Eqn. 10 was used to fit calculated surface pressure values at various interlayer distances for the bilayer structures using the four exchange-correlation functionals to obtain the fit parameters given in Table. III. Fits were done for $d$ values ranging from $80 \%$ of the expected equilibrium interlayer distance value to about $105 \%$ as these gave good pressure ranges for the fits. Plots of the EOS fits are given in Figures $2 b, 2 c$ and $2 d$. A comparison of the shapes of these plots for a given structure gives an indication of how each functional describes the interlayer interaction differently. For example, in plots $2 b$ and $2 d$ there is no discernible minimum for the PBE functional which points to the minimal interlayer interaction within this scheme.

The results in Table. III show the equilibrium interlayer distances obtained from the EOS fits. The fitted values for bilayer graphene using the three non-local functionals are in very good agreement with those of Hamada and Otani who calculated the following distances by minimizing the interlayer binding energy as a function of $d:^{57} 3.581 \AA$ (vdWDF), $3.226 \AA$ (vdW-DF-C09x $)$ and 3.277 $\AA$ (vdW-DF2-C09 $x$ ).
We see that these values are the same as those calculated for graphite (with the exception of that for PBE). The reasons for the relative behavior of the four functionals are the same as those given for graphite.

The values for $d_{0}$ for bilayer graphene with $50 \%$ coverage are almost identical to each other and close in value to the single $\mathrm{C}-\mathrm{C}$ bond found in cubic diamond indicating that the interlayer bonding between opposing carbon atoms is diamondlike covalent. This short-range interaction is similarly described by the differing functionals as was seen in the case for the in-plane covalent bonding in graphene and bilayer graphene.

The values for $d_{0}$ for bilayer graphane are the largest of the bilayer structures due to the fact that the interlayer hydrogen atoms tend to force the layers apart.

The plots for bilayer graphene in Fig. 2b show that GGAPBE predicts the lowest surface pressures for each compression followed in increasing order by vdW-DF $\rightarrow \mathrm{vdW}-\mathrm{DF} 2-$ $\mathrm{C} 09_{x} \rightarrow \mathrm{vdW}-\mathrm{DF}-\mathrm{C} 09_{x}$. This is reflected in Table. III where the value of the interlayer modulus follows the same trend. This is the same trend observed in the calculation of the linear bulk modulus $\left(C_{33}\right)$ for graphite. The interlayer modulus for GGA-PBE is well below those for the three non-local functionals due to its extreme underbinding of the layers caused by its relatively flat interaction potential. The values for the other three functionals give a quantitative comparison of the non-local corrected functionals used. Although vdW-DF and vdW-DF-C09 both contain the non-local correlation term of Dion et al., ${ }^{37}$ they do not have comparable interlayer modulus values. The values show that the $\mathrm{C} 09_{x}$ exchange term in vdW-DF-C09 $x$ enhances the repulsive interlayer interaction as the layers are compressed to a greater extent than the revPBE term in vdW-DF. The non-local corrected functional vdW$\mathrm{DF} 2-\mathrm{C} 09_{x}$ has the non-local term of Lee et al. ${ }^{38}$ and shows a similarly shaped plot to vdW-DF-C09 $x$ as indicated by the similar values for $\beta_{0}^{\prime}$ and $\beta_{0}^{\prime \prime}$ in Table III. It has a lower interlayer modulus than vdW-DF-C09 $x$ indicating that its nonlocal term describes a slightly lower repulsive interlayer interaction during compression.

Also shown in Fig. 2b are minima for the three non-local functions indicating, as in the case for the 2D in-plane fits, the intrinsic breaking strengths at a given negative value for $p$ (an expansion). These values are also given in Table III and show that the structure described by vdW-DF-C09 $x$ requires the highest negative surface pressure to separate the layers. The other two values are comparable in strength. These values indicate that the layers can be more easily separated. As expected, GGA-PBE has no minima due to the lack of adequate interlayer interaction.

The plots for $50 \%$ hydrogenated bilayer graphene in Fig. 2c show that all four functionals give very similar out-of-plane behavior which is reflected in the values in Table III. It is clear that the values for the interlayer modulus are $\sim 10$ times higher in magnitude than those for pristine bilayer graphene ( $\sim 50$ in the case of GGA-PBE). The reason for these high interlayer modulus values is due to the fact that these functionals have very similar short-range interaction potentials. They give a quantitative measure of just how weak the interlayer 
interactions described by the non-local corrected functionals in bilayer graphene are as compared to the covalent interlayer interaction described in bilayer graphene with $50 \%$ coverage. This is due to the fact that for this structure, the reduced interlayer distance causes the non-local term in vdW corrected functionals to effectively vanish leaving only the effects of the LDA correlation term and the exchange terms. These terms yield very similar values for the interlayer modulus for the diamond-like structure of bilayer graphene $+\mathrm{H}(50 \%)$ with a slight increasing trend of vdW-DF $\rightarrow$ GGA-PBE $\rightarrow$ vdW-DF2$\mathrm{C} 09_{x} \rightarrow \mathrm{vdW}-\mathrm{DF}-\mathrm{C} 09_{x}$. As is expected, the values for the two functionals containing the $\mathrm{C} 09_{x}$ exchange term yield almost the same fit parameters.

Fig. 2c also shows minima in all four cases indicative of layer breaking at negative $p$. These values follow the same trend as the interlayer modulus and reveal that the layers for bilayer graphene $+\mathrm{H}(50 \%)$ are not easily separated.

The plots for bilayer graphane in Fig. $2 \mathrm{~d}$ are similar to those for pristine bilayer graphene. Although the surface pressure range is lower for the hydrogenated structure, they share the same general shape with GGA-PBE predicting the lowest pressures for each compression with the same asymptotic behavior. The other three functionals show an increasing trend in the calculated compression pressures from vdW-DF $\rightarrow \mathrm{vdW}-$ DF2-C09 $x \rightarrow$ vdW-DF-C09 $x$. The values for the interlayer modulus, shown in Table III follow the same trend. These values are of the same order of magnitude to those of bilayer graphene indicating similar repulsive interlayer interaction although this interaction is due to the fact that when the hydrogenated structure is compressed vertically, the hydrogen atoms within the interlayer spacing repel each other.

The plots in Fig. 2d for the three non-local corrected functionals show perceptible minima indicative of layer breaking. GGA-PBE has a barely perceptible minima at $d_{\text {int }} / d_{0}=1.169$ as shown in Table III and as such, is not considered significant. The values for $p_{i n t}$ are of the same order of magnitude as bilayer graphene and show that the layers are easily separated.

The binding energy per chemical unit was also calculated for each bilayered structure as follows

$$
E_{b}=\frac{E_{\text {bilayer }}-2 E_{\text {layer }}}{n}
$$

where $n$ is the number of chemical units. The results for the three van der Waals corrected functionals show that for each structure, the trend in the calculated values mirrors the trend for the interlayer modulus values. This shows that how differing functionals describe the out-of-plane mechanical properties, also gives a prediction in how they will relatively predict the binding energies. As with graphite, vdW-DF and vdW-
DF2-C09 $x$ give very similar values for bilayer graphene.

\section{CONCLUSIONS}

We present a consistent theoretical framework for the inplane layer modulus and the out-of-plane interlayer modulus and we calculate, for the first time, these properties for graphene and hydrogenated graphene monolayer and bilayer systems. This gives a measure of the change of the strength properties when pristine graphene is hydrogenated. We test and compare the set of recently developed non-local corrected exchange correlation functionals since they are known to better describe the interlayer interaction.

We find that for all four functionals, the bilayer structures have greater resilience to in-plane stretching than the monolayer structures as indicated by their relatively higher values for $\gamma_{0}$. The hydrogenated structures are also less resilient than their pristine counterparts.

GGA-PBE predicts the lowest surface pressures for each compression followed in increasing order by vdW-DF $\rightarrow \mathrm{vdW}-$ $\mathrm{DF} 2-\mathrm{C} 09_{x} \rightarrow \mathrm{vdW}-\mathrm{DF}-\mathrm{C} 09_{x}$. Consequently, the value of the interlayer modulus follows the same trend. This trend is also observed for graphite. The interlayer modulus for GGA-PBE is well below those for the three non-local functionals due to its extreme underbinding of the layers caused by its relatively flat interaction potential.

The intrinsic breaking strengths show that the structure described by vdW-DF-C09 requires the highest negative surface pressure to separate the layers. Our results indicate that the layers are predicted to be more easily separated using the other two non-local functionals.

For the bilayered materials, it was found that the binding energies follow the same trend as the interlayer modulus values showing a correlation between the two properties.

The calculations for graphite show that of the three van der Waals corrected functionals considered, vdW-DF2-C09x gives consistent accurate results for the interlayer spacing, the binding energy and the elastic constant $C_{33}$. This suggests that it may be the best candidate to use for layered graphene systems.

\section{ACKNOWLEDGMENTS}

We are grateful to the University of Pretoria for the use of computational resources. REM acknowledges the NRF for financial support. RCA and NC thank the National Institute for Theoretical Physics for financial support.
* Richard.Andrew@up.ac.za
+ Edwin.Mapasha@up.ac.za
$\div$ Nithaya.Chetty@up.ac.za
1 K. S. Novoselov, A. K. Geim, S. V. Morozov, D. Jiang, Y. Zhang,
S. V. Dubonos, I. V. Grigorieva, and A. A. Firsov, Science 306,
666 (2004)

2 A. K. Geim and K. S. Novoselov, Nature Mater. 6, 183 (2007).

3 P. R. Wallace, Phys. Rev. 71, 622 (1947).

4 K. S. Novoselov, A. K. Geim, S. V. Morozov, D. Jiang, Y. Zhang, M. I. Katsnelson, S. V. Dubonos, I. V. Grigorieva, and A. A. 
Firsov, Nature 438, 197 (2005).

${ }^{5}$ K. S. Novoselov, Z. Jiang, Y. Zhang, S. V. Morozov, H. L. Stormer, U. Zeitler, J. C. Maan, G. S. Boebinger, P. Kim, and A. K. Geim, Science 315, 1379 (2007).

${ }^{6}$ Y. Zhang, Y. W. Tan, H. L. Stormer, and P. Kim, Nature 438, 201 (2005).

7 A. H. Castro, F. Guinea, N. M. R. Peres, K. S. Novoselov, and A. K. Geim, Rev. Mod. Phys. 81, 109 (2009).

${ }^{8}$ F. Schedin, A. K. Geim, S. V. Morozov, E. W. Hill, P. Blake, M. I. Katsnelson, and K. S. Novoselov, Nature Mater. 6, 652 (2007).

9 T. Ohta, A. Bostwick, T. Seyller, K. Horn, and E. Rotenberg, Science 313, 951 (2006).

10 Y. H. Lu, W. Chen, Y. P. Feng, and P. M. He, J. Phys. Chem. B 113, 2 (2009).

11 I. Zanella, S. Guerini, S. B. Fagan, J. MendesFilho, and A. G. SouzaFilho, Phys. Rev. B 77, 073404 (2008).

12 R. M. Ribeiro, N. M. R. Peres, J. Coutinho, and P. R. Briddon, Phys. Rev. B 78, 075442 (2008).

13 D. W. Boukhvalov, M. I. Katsnelson, and A. I. Lichtenstein, Phys. Rev. B 77, 035427 (2008).

${ }^{14}$ S. Lebegue, M. Klintenberg, O. Eriksson, and M. I. Katsnelson, Phys. Rev. B 79, 245117 (2009).

15 E. J. Duplock, M. Scheffler, and P. J. D. Lindan, Phys. Rev. Lett. 92, 225502 (2004)

${ }^{16}$ D. W. Boukhvalov and M. I. Katsnelson, Phys. Rev. B 78, 085413 (2008).

17 N. Z. Lu, Z. Y. Li, and J. L. Yang, J. Phys. Chem. C 113, 16741 (2009).

18 J. A. Yan, L. Xian, and M. Y. Chou, Phys. Rev. Lett. 103, 086802 (2009).

${ }^{19}$ X. S. Wu, M. Sprinkle, X. B. Li, F. Ming, C. Berger, and W. A. de Heer, Phys. Rev. Lett. 101, 026801 (2008).

${ }^{20}$ I. Jung, D. A. Dikin, R. D. Piner, and R. S. Ruoff, Nano Lett. 8, $4283(2008)$

21 Z. Luo, P. M. Vora, E. J. Mele, A. T. . C. Johnson, and J. M. Kikkawa, Appl. Phys. Lett. 94, 111909 (2009).

${ }^{22}$ R. Balog, B. Jorgensen, L. Nilsson, M. Andersen, E. Rienks, M. Bianchi, M. Fanetti, E. Laegsgaard, A. Baraldi, S. Lizzit, Z. Sljivancanin, F. Besenbacher, B. Hammer, T. G. Pedersen, P. Hofmann, and L. Hornekaer, Nature Mater. 9, 315 (2010).

23 A. Savchenko, Science 323, 589 (2009).

24 J. O. Sofo, A. S. Chaudhari, and G. D. Barber, Phys. Rev. B 75, 153401 (2007).

25 D. C. Elias, R. R. Nair, T. M. G. Mohiuddin, S. V. Morozov, P. Blake, M. P. Halsall, A. C. Ferrari, D. W. Boukhvalov, M. I. Katsnelson, A. K. Geim, and K. S. Novoselov, Science 323, 610 (2009).

${ }^{26}$ K. R. Kganyago and P. E. Ngoepe, Phys. Rev. B 68, 205111 (2003).

${ }^{27}$ M. Khantha, N. A. Cordero, L. M. Molina, J. A. Alonso, and L. A. Girifalco, Phys. Rev. B 70, 125422 (2004)

${ }^{28}$ K. T. Chan, J. B. Neaton, and M. L. Cohen, Phys. Rev. B 77, 235430 (2008).

29 R. E. Mapasha and N. Chetty, Comput. Mat. Sci. 49, 787 (2010).

${ }^{30}$ K. Sugawara, K. Kanetani, T. Sato, and T. Takahashi, AIP. Adv. 1, 022103 (2011).
31 A. M. Garay-Tapia, A. H. Romero, and V. Barone, J. Chem. Theory Comput. 8, 1064 (2012).

32 O. Leenaerts, B. Partoens, and F. M. Peeters, Phys. Rev. B 80, 245422 (2009).

33 J. Rohrer and P. Hyldgaard, Phys. Rev. B 83, 165423 (2011).

${ }^{34}$ R. E. Mapasha, A. M. Ukpong, and N. Chetty, Physical Review B 85, 205402 (2012).

35 R. C. Andrew, R. E. Mapasha, A. M. Ukpong, and N. Chetty, Physical Review B 85, 125428 (2012).

36 S. Grimme, J Comput Chem 27, 1787 (2006).

37 M. Dion, H. Rydberg, E. Schröder, D. C. Langreth, and B. I. Lundqvist, Physical Review Letters 92, 246401 (2004).

${ }^{38}$ K. Lee, E. D. Murray, L. Kong, B. I. Lundqvist, and D. C. Langreth, Physical Review B 82, 081101 (2010).

39 Y. Zhang and W. Yang, Phys. Rev. Lett. 80, 890 (1998).

40 V. R. Cooper, Physical Review B 81, 161104 (2010).

41 J. F. Dobson, A. White, and A. Rubio, Physical Review Letters 96, 073201 (2006).

${ }^{42}$ D. C. Langreth and J. P. Perdew, Physical Review B 15, 2884 (1977).

43 S. Lebègue, J. Harl, T. Gould, J. G. Ángyán, G. Kresse, and J. F. Dobson, Physical Review Letters 105, 196401 (2010).

44 T. Björkman, A. Gulans, a. V. Krasheninnikov, and R. M. Nieminen, Journal of physics. Condensed matter 24, 424218 (2012).

45 J. P. Perdew, K. Burke, and M. Ernzerhof, Physical Review Letters 77, 3865 (1996).

46 T. Thonhauser, V. R. Cooper, S. Li, A. Puzder, P. Hyldgaard, and D. C. Langreth, Physical Review B 76, 125112 (2007).

47 G. Román-Pérez and J. M. Soler, Physical Review Letters 103, 096102 (2009)

48 M. Hanfland, H. Beister, and K. Syassen, Physical Review B 39, 12598 (1989).

49 P. Hohenberg and W. Kohn, Physical Review 136, 864 (1964).

50 P. E. Blochl, Physical Review B 50, 17953 (1994).

51 P. Giannozzi, S. Baroni, N. Bonini, M. Calandra, R. Car, C. Cavazzoni, D. Ceresoli, G. L. Chiarotti, M. Cococcioni, I. Dabo, A. Dal Corso, S. de Gironcoli, S. Fabris, G. Fratesi, R. Gebauer, U. Gerstmann, C. Gougoussis, A. Kokalj, M. Lazzeri, L. Martin-Samos, N. Marzari, F. Mauri, R. Mazzarello, S. Paolini, A. Pasquarello, L. Paulatto, C. Sbraccia, S. Scandolo, G. Sclauzero, A. P. Seitsonen, A. Smogunov, P. Umari, and R. M. Wentzcovitch, Journal of Physics: Condensed Matter 21, 395502 (2009).

52 H. J. Monkhorst and J. D. Pack, Physical Review B 13, 5188 (1976).

${ }^{53}$ M. Methfessel and A. T. Paxton, Physical Review B 40, 3616 (1989).

54 R. Sabatini, E. Küçükbenli, B. Kolb, T. Thonhauser, and S. de Gironcoli, Journal of physics: Condensed matter 24, 424209 (2012).

55 A. Bosak, M. Krisch, M. Mohr, J. Maultzsch, and C. Thomsen, Physical Review B 75, 153408 (2007).

56 R. Zacharia, H. Ulbricht, and T. Hertel, Physical Review B 69, 155406 (2004).

57 see supplemental material for I. Hamada and M. Otani, Physical Review B 82, 153412 (2010) as found at http://link.aps . org/supplemental/10.1103/PhysRevB.82.153412 


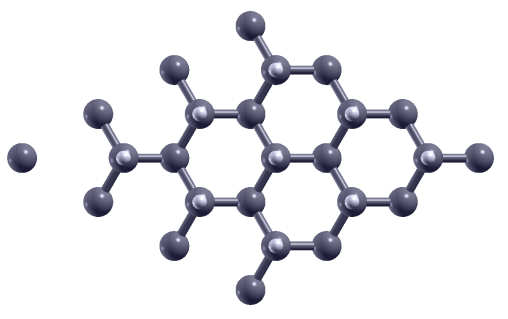

(a)

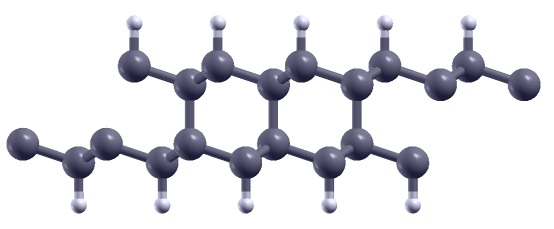

(c)

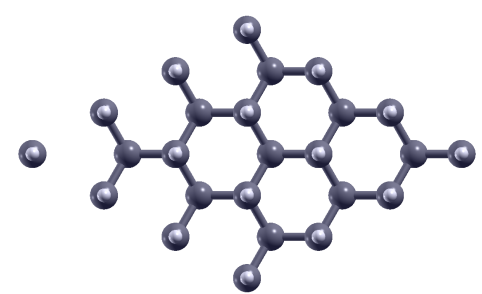

(b)

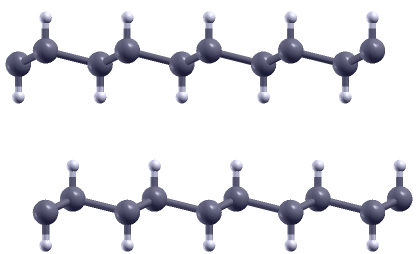

(d)

FIG. $1.3 \times 3$ cells of the four hydrogenated structures: (a) top-view of monolayer graphene with $50 \%$ hydrogen coverage, (b) bottom-view of graphane with $100 \%$ hydrogen coverage, (c) side-view of bilayer graphene with $50 \%$ hydrogen coverage, and (d) side-view of bilayer graphane with $100 \%$ hydrogen coverage. (hydrogen atoms $\bigcirc$ carbon atoms $\bullet$ ) 


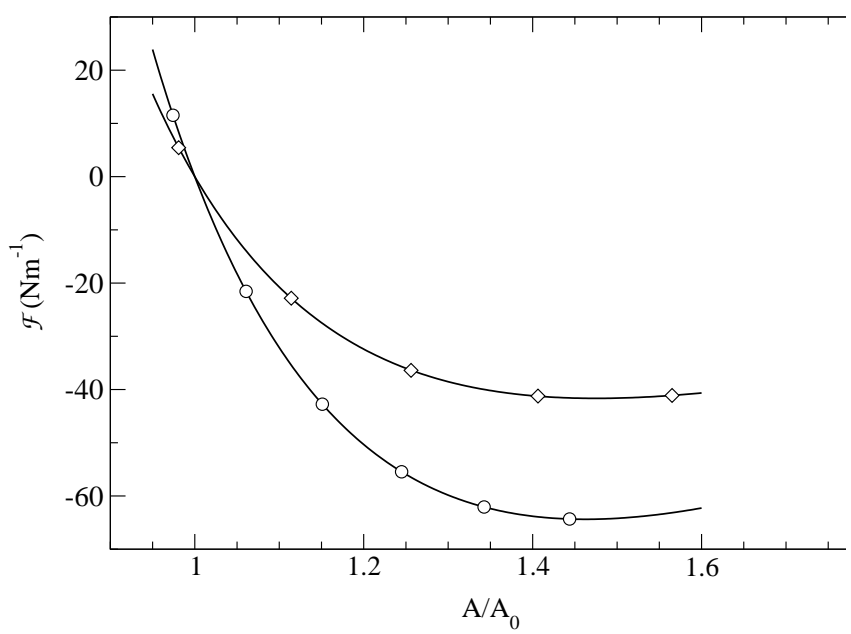

(a)

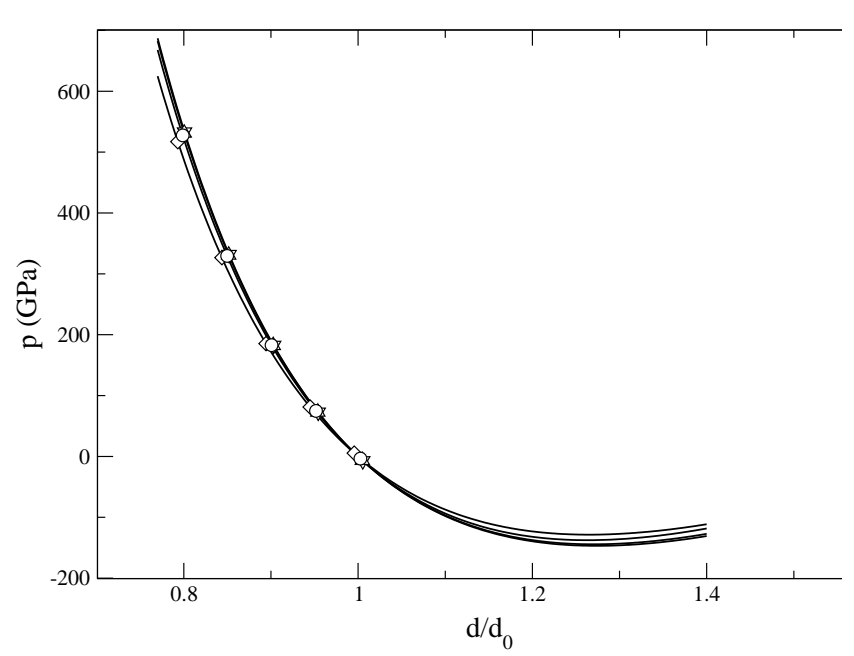

(c)

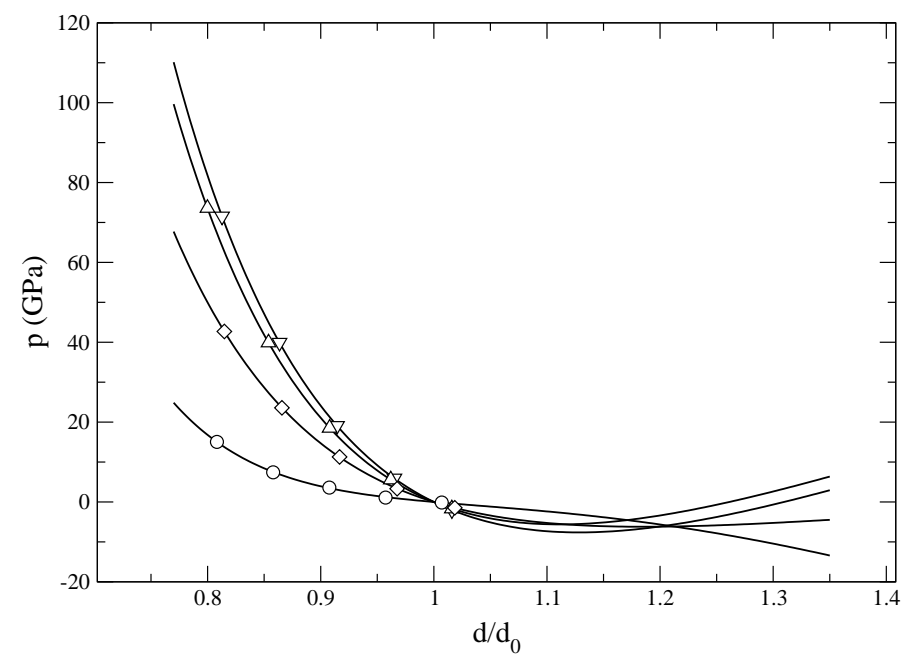

(b)

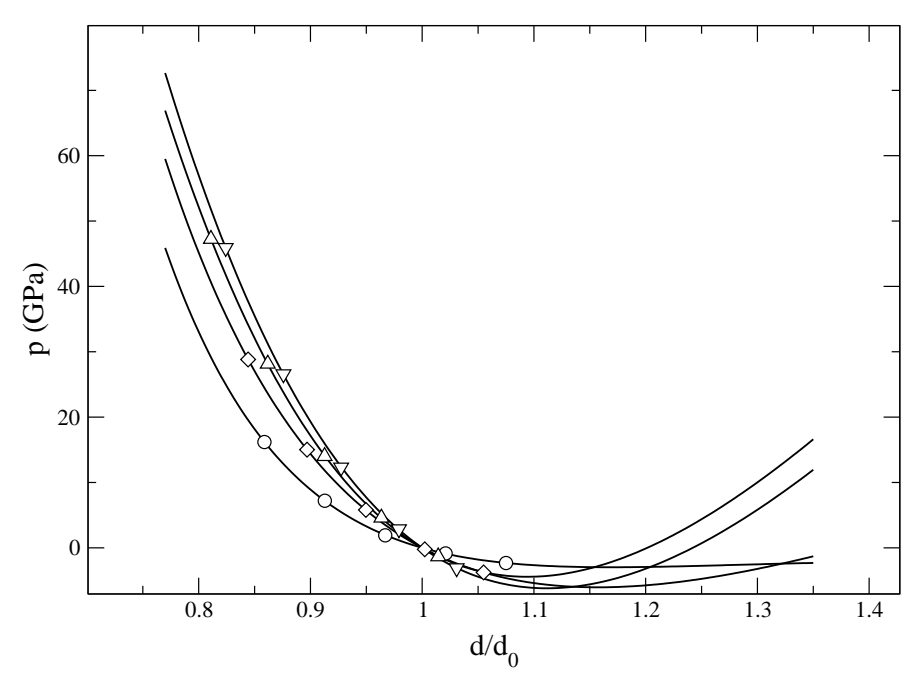

(d)

FIG. 2. EOS fit plots showing (a) the in-plane 2D pressure $\mathcal{F}(A)$ equation of state fits for bilayer graphene $(\circ)$ and bilayer graphane $(\diamond)$ using the GGA-PBE functional with the plot showing minima for the expected intrinsic breaking points, (b) the out-of-plane equation of state fits for bilayer graphene for all four functionals (० PBE, $\diamond \mathrm{vdW}-\mathrm{DF}, \nabla \mathrm{vdW}-\mathrm{DF}-\mathrm{C} 09_{x}$ and $\Delta \mathrm{vdW}-\mathrm{DF} 2-\mathrm{C} 09_{x}$ ) showing minima for the three non-local corrected functionals, (c) the out-of-plane equation of state fits for bilayer graphene with $50 \%$ hydrogen coverage for all four functionals ( $\circ \mathrm{PBE}, \diamond \mathrm{vdW}-\mathrm{DF}, \nabla \mathrm{vdW}-\mathrm{DF}-\mathrm{C} 09_{x}$ and $\Delta \mathrm{vdW}-\mathrm{DF} 2-\mathrm{C} 09_{x}$ ) showing minima in all four cases and (d) the out-of-plane equation of state fits for bilayer graphane for all four functionals (० PBE, $\diamond \mathrm{vdW}-\mathrm{DF}, \nabla \mathrm{vdW}-\mathrm{DF}-\mathrm{C} 09_{x}$ and $\Delta \mathrm{vdW}-\mathrm{DF} 2-$ $\mathrm{C} 09_{x}$ ) showing perceptible minima for the last three functionals. 
TABLE I. In-plane structural and mechanical properties for pristine and hydrogenated single and bi-layered graphene (lattice constant $a_{0}$ and buckling parameter $\Delta$ in $\AA$, layer modulus $\gamma_{0}$ in $\mathrm{N} \mathrm{m}^{-1}, \gamma_{0}^{\prime}$ (dimensionless), $\gamma_{0}^{\prime \prime}$ in $\mathrm{m} \mathrm{N}^{-1}$, relative area at failure $A_{\text {int }} / A_{0}$ (dimensionless), and stress at failure $\sigma_{\text {int }}$ in $\mathrm{N} \mathrm{m}^{-1}$ ).

\begin{tabular}{|c|c|c|c|c|c|c|c|c|}
\hline & & $a_{0}$ & $\Delta$ & $\gamma_{0}$ & $\gamma_{0}^{\prime}$ & $\gamma_{0}^{\prime \prime}$ & $A_{\text {int }} / A_{0}$ & $\sigma_{\text {int }}$ \\
\hline \multirow[t]{4}{*}{ graphene } & GGA-PBE & 2.47 & & 208.2 & 4.38 & -0.0320 & 1.46 & 32.2 \\
\hline & vdW-DF & 2.48 & & 202.1 & 4.49 & -0.0342 & 1.45 & 30.5 \\
\hline & vdW-DF-C09 $9_{X}$ & 2.46 & & 210.3 & 4.38 & -0.0314 & 1.46 & 32.5 \\
\hline & $\mathrm{vdW}-\mathrm{DF} 2-\mathrm{C} 09_{X}$ & 2.46 & & 209.2 & 4.40 & -0.0318 & 1.46 & 32.3 \\
\hline \multirow[t]{4}{*}{ bilayer graphene } & GGA-PBE & 2.47 & & 417.2 & 4.40 & -0.0159 & 1.46 & 64.3 \\
\hline & vdW-DF & 2.48 & & 403.8 & 4.49 & -0.0172 & 1.45 & 61.0 \\
\hline & vdW-DF-C09 $X$ & 2.46 & & 420.6 & 4.39 & -0.0157 & 1.46 & 65.0 \\
\hline & vdW-DF2-C09 & 2.46 & & 418.7 & 4.40 & -0.0159 & 1.46 & 64.6 \\
\hline \multirow[t]{4}{*}{ graphene $+\mathrm{H}(50 \%)$} & GGA-PBE & 2.53 & 0.26 & 139.5 & 3.68 & -0.0619 & 1.43 & 22.4 \\
\hline & vdW-DF & 2.54 & 0.28 & 135.7 & 4.15 & -0.0583 & 1.43 & 20.8 \\
\hline & vdW-DF-C09 $x$ & 2.52 & 0.26 & 142.7 & 3.74 & -0.0569 & 1.44 & 23.1 \\
\hline & vdW-DF2-C09 & 2.52 & 0.26 & 141.6 & 3.77 & -0.0576 & 1.44 & 22.8 \\
\hline \multirow[t]{4}{*}{ graphane } & GGA-PBE & 2.54 & 0.46 & 135.2 & 4.48 & -0.0468 & 1.47 & 20.9 \\
\hline & vdW-DF & 2.56 & 0.46 & 129.7 & 4.58 & -0.0513 & 1.46 & 19.6 \\
\hline & vdW-DF-C09 & 2.53 & 0.46 & 139.6 & 4.47 & -0.0449 & 1.48 & 21.6 \\
\hline & vdW-DF2-C09 & 2.53 & 0.46 & 138.2 & 4.50 & -0.0458 & 1.47 & 21.3 \\
\hline \multirow[t]{4}{*}{ bilayer graphene $+\mathrm{H}(50 \%)$} & GGA-PBE & 2.53 & 0.49 & 264.0 & 4.52 & -0.0252 & 1.46 & 40.1 \\
\hline & vdW-DF & 2.55 & 0.49 & 253.2 & 4.64 & -0.0277 & 1.45 & 37.5 \\
\hline & vdW-DF-C09 $x$ & 2.52 & 0.49 & 271.6 & 4.51 & -0.0241 & 1.47 & 41.5 \\
\hline & $\mathrm{vdW}-\mathrm{DF} 2-\mathrm{C} 09_{X}$ & 2.52 & 0.49 & 269.0 & 4.50 & -0.0245 & 1.46 & 41.0 \\
\hline \multirow[t]{4}{*}{ bilayer graphane } & GGA-PBE & 2.54 & 0.46 & 270.5 & 4.51 & -0.0233 & 1.47 & 41.7 \\
\hline & vdW-DF & 2.56 & 0.47 & 258.9 & 4.61 & -0.0258 & 1.46 & 38.9 \\
\hline & vdW-DF-C $09_{X}$ & 2.53 & 0.46 & 279.0 & 4.52 & -0.0227 & 1.47 & 42.9 \\
\hline & vdW-DF2-C09 & 2.53 & 0.46 & 276.1 & 4.51 & -0.0230 & 1.47 & 42.4 \\
\hline
\end{tabular}


TABLE II. Ratios of material in-plane EOS fit parameters to compare the effects of hydrogenation and layering with the corresponding reference structure.

\begin{tabular}{|c|c|c|c|c|c|c|c|}
\hline & & $a_{0}$ & $\gamma_{0}$ & $\gamma_{0}^{\prime}$ & $\gamma_{0}^{\prime \prime}$ & $A_{\text {int }}$ & $\sigma_{i n t}$ \\
\hline bilayer graphene & GGA-PBE & 1.00 & 2.00 & 1.00 & 0.50 & 1.00 & 2.00 \\
\hline \multirow[t]{3}{*}{ compared with graphene } & vdW-DF & 1.00 & 2.00 & 1.00 & 0.50 & 1.00 & 2.00 \\
\hline & vdW-DF-C $09_{X}$ & 1.00 & 2.00 & 1.00 & 0.50 & 1.00 & 2.00 \\
\hline & vdW-DF2-C09 ${ }_{X}$ & 1.00 & 2.00 & 1.00 & 0.50 & 1.00 & 2.00 \\
\hline graphene $+\mathrm{H}(50 \%)$ & GGA-PBE & 1.03 & 0.67 & 0.84 & 1.93 & 1.03 & 0.70 \\
\hline \multirow[t]{3}{*}{ compared with graphene } & vdW-DF & 1.03 & 0.67 & 0.92 & 1.70 & 1.04 & 0.68 \\
\hline & vdW-DF-C09 $X$ & 1.02 & 0.68 & 0.85 & 1.81 & 1.03 & 0.71 \\
\hline & vdW-DF2-C09 ${ }_{X}$ & 1.02 & 0.68 & 0.86 & 1.81 & 1.03 & 0.71 \\
\hline graphane & GGA-PBE & 1.03 & 0.65 & 1.02 & 1.46 & 1.07 & 0.65 \\
\hline \multirow[t]{3}{*}{ compared with graphene } & vdW-DF & 1.03 & 0.64 & 1.02 & 1.50 & 1.07 & 0.64 \\
\hline & vdW-DF-C09 $x$ & 1.03 & 0.66 & 1.02 & 1.43 & 1.06 & 0.66 \\
\hline & vdW-DF2-C09 ${ }_{X}$ & 1.03 & 0.66 & 1.02 & 1.44 & 1.06 & 0.66 \\
\hline bilayer graphene $+\mathrm{H}(50 \%)$ & GGA-PBE & 1.00 & 1.89 & 1.23 & 0.41 & 1.02 & 1.79 \\
\hline \multirow[t]{3}{*}{ compared with graphene $+\mathrm{H}(50 \%)$} & vdW-DF & 1.00 & 1.87 & 1.12 & 0.48 & 1.01 & 1.80 \\
\hline & $\mathrm{vdW}-\mathrm{DF}-\mathrm{C} 09_{X}$ & 1.00 & 1.90 & 1.21 & 0.42 & 1.02 & 1.80 \\
\hline & vdW-DF2-C09 ${ }_{X}$ & 1.00 & 1.90 & 1.19 & 0.43 & 1.02 & 1.80 \\
\hline bilayer graphane & GGA-PBE & 1.00 & 2.00 & 1.01 & 0.50 & 1.00 & 2.00 \\
\hline \multirow[t]{3}{*}{ compared with graphane } & vdW-DF & 1.00 & 2.00 & 1.01 & 0.50 & 1.00 & 1.98 \\
\hline & vdW-DF-C09 ${ }_{X}$ & 1.00 & 2.00 & 1.01 & 0.51 & 1.00 & 1.99 \\
\hline & vdW-DF2-C09 & 1.00 & 2.00 & 0.99 & 0.50 & 1.00 & 1.99 \\
\hline
\end{tabular}

TABLE III. Out-of-plane structural and mechanical properties for the bi-layered structures (interlayer distance $d_{0}$ in $\AA$, interlayer modulus $\beta_{0}$ in $\mathrm{GPa}, \beta_{0}^{\prime}$ (dimensionless), $\beta_{0}^{\prime \prime}$ in $\mathrm{GPa}^{-1}$, relative interlayer distance at failure $d_{\text {int }} / d_{0}$ (dimensionless), surface pressure at failure $p_{\text {int }}$ in GPa and binding energy per chemical unit $E_{b}$ in $\mathrm{meV}$ ).

\begin{tabular}{|c|c|c|c|c|c|c|c|c|}
\hline & & $d_{0}$ & $\beta_{0}$ & $\beta_{0}^{\prime}$ & $\beta_{0}^{\prime \prime}$ & $d_{\text {int }} / d_{0}$ & $p_{\text {int }}$ & $E_{b}$ \\
\hline \multirow{5}{*}{ graphite } & GGA-PBE & 4.12 & $5.1^{a}$ & $9.96^{b}$ & & & & $<1$ \\
\hline & vdW-DF & 3.59 & $27.1^{a}$ & $9.19^{b}$ & & & & 56 \\
\hline & vdW-DF-C09 $X$ & 3.24 & $45.1^{a}$ & $10.4^{b}$ & & & & 76 \\
\hline & vdW-DF2-C09 & 3.28 & $38.3^{a}$ & $10.9^{b}$ & & & & 55 \\
\hline & Experimental & 3.356 & $38.7^{c}$ & & & & & $52^{d}$ \\
\hline \multirow[t]{4}{*}{ bilayer graphene } & GGA-PBE & 3.99 & 24.8 & 3.84 & 5.009 & & & $\sim 1$ \\
\hline & vdW-DF & 3.59 & 87.8 & 9.24 & -0.410 & 1.185 & -6.2 & 26 \\
\hline & vdW-DF-C09 $X$ & 3.23 & 138.5 & 10.64 & -0.493 & 1.128 & -7.6 & 34 \\
\hline & vdW-DF2-C09 ${ }_{X}$ & 3.28 & 115.6 & 11.87 & -0.776 & 1.111 & -5.6 & 25 \\
\hline \multirow[t]{4}{*}{ bilayer graphene + H(50\%) } & GGA-PBE & 1.56 & 1315.3 & 5.74 & -0.0139 & 1.263 & -137.5 & 1920 \\
\hline & vdW-DF & 1.57 & 1225.5 & 5.75 & -0.0147 & 1.265 & -128.5 & 1940 \\
\hline & vdW-DF-C09 $X$ & 1.55 & 1363.7 & 5.62 & -0.0125 & 1.274 & -146.8 & 2120 \\
\hline & vdW-DF2-C09 ${ }_{X}$ & 1.55 & 1352.1 & 5.66 & -0.0129 & 1.270 & -144.3 & 2080 \\
\hline \multirow[t]{4}{*}{ bilayer graphane } & GGA-PBE & 4.48 & 48.6 & 11.10 & -0.986 & $1.169^{e}$ & $-3.0^{e}$ & $\sim 1$ \\
\hline & vdW-DF & 4.29 & 90.2 & 8.66 & -0.517 & 1.157 & -6.0 & 20 \\
\hline & vdW-DF-C09 ${ }_{X}$ & 4.02 & 119.3 & 9.95 & -0.765 & 1.112 & -6.2 & 26 \\
\hline & vdW-DF2-C09 & 4.08 & 100.2 & 11.61 & -1.25 & 1.094 & -4.4 & 17 \\
\hline
\end{tabular}

${ }^{a} B_{c}$ for graphite which is equivalent to $C_{33}$

${ }^{b} B_{c}^{\prime}$ for graphite

${ }^{c} C_{33}$ for graphite determined by inelastic x-ray scattering. Bosak et al. ${ }^{55}$

${ }^{d}$ for graphite determined from thermal desorption studies. Zacharia et al. ${ }^{56}$

${ }^{e}$ not significant 